\title{
The groups of Poincaré and Galilei in arbitrary dimensional spaces
}

\author{
E. Elizalde ${ }^{\text {a) }}$ and J. Gomis
}

Department de Fisica Teórica, Universitat de Barcelona, Diogonal 647, Barcelona-14. Spain

(Received 19 September 1977)

\begin{abstract}
In arbitrary dimensional spaces the Lie algebra of the Poincare group is seen to be a subalgebra of the complex Galilei algebra, while the Galilei algebra is a subalgebra of Poincaré algebra. The usual contraction of the Poincaré to the Galitei group is seen to be equivalent to a certain coordinate transformation.
\end{abstract}

\section{INTRODUCTION}

It is well known that the Galilei group in $2+1$ dimensions is a subgroup of that of Poincaré. A beautiful way to see this is by means of a change of coordinates ${ }^{1}$ which is usually called the light-cone transformation. This fact is physically understood as the result of an infinite boost of a system in some direction of the space, which leads to the loss of the spatial dimension in this direction-due to the Lorentz contraction-and leaves the remaining system with a Galilean structure. This method has had several applications, for instance, to the study of the internal structure of hadrons at very high energies ${ }^{2}$ and to the connection of relativistic and Galilean field equations for arbitrary spin particle. ${ }^{3}$ Moreover, with a modification of the light-cone transformation involving a continuous parameter ${ }^{4}$ it has been possible to go from the $(2+1)$-dimensional Galilei group to the Poincaré one in the same dimension, in a procedure inverse to the ordinary contraction of the Poincare to the Galilei group when $c \rightarrow \infty$.

On the other hand, it has recently been shown that the ordinary Lie algebra of the Poincare group is a subalgebra ${ }^{5}$ of the complex Lie algebra of the Galilei group in $4+1$ dimensions. Also this result has been obtained through an adequate change of coordinates. Nevertheless its physical interpretation is not so clear. One possible application of this connection is the derivation of relativistic equations starting with Galilean ones, in just the reciprocal way of the former case. ${ }^{6}$

Summing up, the complex Galilei algebra in $4+1$ dimensions contains the ordinary Poincaré algebra, which in turn contains a $(2+1)$-dimensional Galilei algebra, and these relations have their parallel counterpart at the level of the corresponding wave equations. The generalization of this situation to an arbitrary number $n$ of space dimensions is one of the objects of the present paper. In this way we shall see that for an abstract physical theory the election of one or another invariance group (i.e., Poincaré or Galilei) is not so fundamental as one would think, because it is possible to obtain a relativistic theory in $(n-1)+1$ dimensions from a Galilean one in $n+1$ dimensions, and vice-versa.

Another purpose of this work is to investigate which is in the present case the modification of the coordinate transformation ${ }^{5}$ which enables us to obtain the ordinary Galilei

${ }^{a}$ Address after 1 October 1977: II. Institut für Theoretische Physik der Universität Hamburg. group from the Poincaré one in the same dimension, as well as the relation of this modificated coordinate transformation with the usual contraction of the Poincare to the Galilei group. In other words, we want to study the corresponding procedure to that of the quasi-light-cone frame $\mathrm{e}^{4}$ in the present case.

The organization of the paper is as follows: In Sec. 2, we generalize the coordinate transformation of Ref. 5 and see how the Poincare algebra in $n$ space/ 1 time dimensions is a subalgebra of the complex Galilei algebra in $(n+1)$ space/1 time coordinates. In Sec. 3 we generalize the light-cone frame transformation to an arbitrary number of dimensions of the space. In Sec. 4 we develop a parametrization of the original coordinate transformation which enables us to pass from the Poincare to the Galilei group in the same number of dimensions. We study its relation with the ordinary contraction $c \rightarrow \infty$. Section 5 is devoted to conclusions.

\section{2. $P_{n+1}$ AS A SUBGROUP OF $G_{(n+1)+1}$}

The Lie algebra of the extended Galilei group in $(n+1)$ space $/ 1$ time dimensions is given by

$$
\begin{aligned}
& {\left[L_{r s}, L_{u v}\right]=i\left(\delta_{r v} L_{s u}+\delta_{s u} L_{r v}-\delta_{r u} L_{s v}-\delta_{s v} L_{r u}\right),} \\
& {\left[L_{r s}, G_{u}\right]=-i\left(\delta_{r u} G_{s}-\delta_{s u} G_{r}\right),} \\
& {\left[L_{r s}, P_{u}\right]=-i\left(\delta_{r u} P_{s}-\delta_{s u} P_{r}\right),} \\
& {\left[G_{r}, G_{s}\right]=\left[P_{r}, P_{s}\right]=\left[L_{r s}, H\right]=\left[P_{r}, H\right]=0,} \\
& {\left[G_{r}, H\right]=i P_{r},} \\
& {\left[G_{r} P_{s}\right]=i \delta_{r s} \mu} \\
& \quad(r, s, u, v=1,2, \cdots, n+1), \\
& {\left[L_{r s}, \mu\right]=\left[P_{r} \mu\right]=\left[G_{r}, \mu\right]=[H, \mu]=0,}
\end{aligned}
$$

where $L_{r s}$ are the generators of rotations, $G_{r}$ the generators of Galilean boosts, $P_{r}$ those of the space translations, $H$ the generator of time translations, and $\mu$ is the neutral element of the algebra, which is associated with the mass.

The generalization of the coordinate transformation introduced in Ref. 5 to this case is the following. If $x^{\alpha}=\left(x^{0}, x^{1}, \cdots, x^{n+1}\right)$ are the old and $x^{\alpha} \equiv\left(\bar{x}^{0}, \bar{x}^{1}, \cdots, x^{n+1}\right)$ the new coordinates of an arbitrary point in the space-time- $x^{0}$ being the time coordinate-they are related by

$$
\begin{aligned}
& \bar{x}^{0}=i x^{n+1}, \\
& \bar{x}^{i}=x^{i} \quad(i=1,2 \cdots, n), \\
& \bar{x}^{n+1}=x^{0} \gamma(\gamma \text { arbitrary }) .
\end{aligned}
$$


With this transformation the real Lie algebra (2.1) becomes a complex algebra of Galilei group. Let us consider the following subset of the transformed generators:

$$
\begin{aligned}
& m_{i j} \equiv \overline{-L}_{i j}=-L_{i j}, \\
& K_{i} \equiv{\overline{L^{0 i}}}=-i L_{n+1, i}, \\
& d_{i} \equiv \bar{P}_{i}=P_{i} \quad(i, j+1,2, \ldots, \mu), \\
& h \equiv P_{0}=-i P_{n+1},
\end{aligned}
$$

The reason for the selection of these generators has already been discussed in Ref. 6 . The commutation relations for these generators are the following:

$$
\begin{aligned}
& {\left[m_{i j}, m_{k l}\right]=-i\left(\delta_{i l} m_{j k}+\delta_{j k} m_{i l}-\delta_{i k} m_{j l}-\delta_{j l} m_{i k}\right),} \\
& {\left[m_{i j}, k_{l}\right]=i\left(\delta_{i l} k_{j}-\delta_{j l} k_{i}\right),} \\
& {\left[m_{i j}, d_{l}\right]=i\left(\delta_{i i} d_{j}-\delta_{j} d_{i}\right),} \\
& {\left[k_{i}, k_{j}\right]=-i m_{i j},} \\
& {\left[d_{i}, d_{j}\right]=\left[m_{i j}, h\right]=\left[d_{i}, h\right]=0,} \\
& {\left[k_{i}, h\right]=i d_{i}, \quad\left[k_{i}, d_{j}\right]=i \delta_{i j} h,}
\end{aligned}
$$

which constitute the Lie algebra of the Poincare group in $n$ space/1 time dimensions.

\section{LIGHT-CONE TRANSFORMATION IN ANY NUMBER OF DIMENSIONS}

The commutation relations of the $(n+1)$-dimensional Poincaré group which we have just obtained can be written in the compact form

$$
\begin{aligned}
& {\left[M_{\mu \nu}, M_{\rho \sigma}\right]=i\left(g_{\mu \sigma} M_{\nu \rho}+g_{\nu \rho} M_{\mu \sigma}\right.} \\
& \left.-g_{\mu \rho} M_{v \sigma}-g_{\nu \sigma} M_{\mu \rho}\right) \text {, } \\
& {\left[P_{\mu}, M_{\rho \sigma}\right]=i\left(g_{\mu \rho} P_{\sigma}-g_{\mu \sigma} P_{\rho}\right),} \\
& {\left[P_{\mu}, P_{\nu}\right]=0 \quad(\mu, \nu, \rho, \sigma=0,1, \ldots, n) \text {, }} \\
& l_{a b} \equiv-M_{a b}, \quad h \equiv\left(\bar{P}_{0}+\bar{P}_{n}\right) / \sqrt{2}, \\
& g_{a} \equiv\left(K_{a}-M_{a n}\right) / \sqrt{2}, \quad \eta=\left(\bar{P}_{0}-\bar{P}_{n}\right) / \sqrt{2}, \\
& d_{a} \equiv P_{a}, \quad(a . b=1,2, \cdots, n) .
\end{aligned}
$$$$
\overline{\bar{M}}_{\mu \nu}=\left(\begin{array}{ccc}
0 & -\left(K_{1}+M_{1 n}\right) / \sqrt{2} & -\left(K_{2}+M_{2 n}\right) / \sqrt{2} \\
\left(K_{1}+M_{1 n}\right) / \sqrt{2} & 0 & M_{12} \\
\left(K_{2}+M_{2 n}\right) / \sqrt{2} & -M_{12} & 0 \\
\cdot & \vdots & \vdots \\
-K_{n} & \left(-K_{1}+M_{1 n}\right) / \sqrt{2} & \left(-K_{2}+M_{2 n}\right) / \sqrt{2}
\end{array}\right.
$$

They satisfy the following commutation relations:

$$
\begin{aligned}
& {\left[l_{a b}, l_{c d}\right]=i\left(\delta_{a d} l_{b c}+\delta_{b c} l_{a d}-\delta_{a c} l_{b d}-\delta_{b d} l_{a c}\right),} \\
& {\left[l_{a b}, g_{c}\right]=-i\left(\delta_{a c} g_{b}-\delta_{b o} g_{a}\right),} \\
& {\left[l_{a b}, d_{c}\right]=-i\left(\delta_{a c} d_{b}-\delta_{b c} d_{a}\right),} \\
& {\left[g_{a}, g_{b}\right]=\left[d_{a}, d_{b}\right]=\left[l_{a b}, h\right]=\left[d_{a}, h\right]=0,} \\
& {\left[g_{a}, h\right]=i d_{a}, \quad\left[g_{a}, d_{b}\right]=i \delta_{a b} \eta,} \\
& {\left[l_{a b}, \eta\right]=\left[d_{a}, \eta\right]=\left[g_{a}, \eta\right]=[h, \eta]=0} \\
& (a, b, c, d=1,2, \ldots, n-1),
\end{aligned}
$$

where $K_{i}=M_{i 0}(i=1,2, \cdots, n)$ are the generators of boosts, $M_{i j}$ $(i, j=1,2, \cdots, n)$ those of the rotations, $P_{i}$ those of the space translations, and $H=\vec{P}_{0}$ the generator of the time translations. The metric tensor is

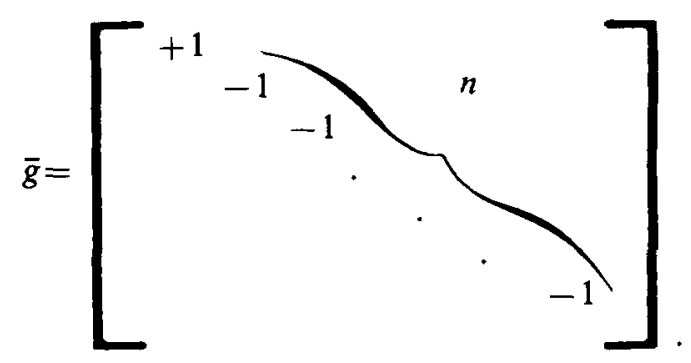

The generalization of the ordinary light-cone frame transformation to the present case is

$$
\begin{aligned}
& \bar{x}^{0}=\left(\bar{x}_{0}+\bar{x}_{n}\right) \sim \sqrt{2}, \\
& \bar{x}^{a}=\bar{x}^{a} \quad(a=1,2, \cdots, n-1) \\
& \bar{x}^{n}=\left(\bar{x}_{0}-\bar{x}_{n}\right) / \sqrt{2}, \\
& \bar{x}^{n+1}=\bar{x}^{n+1} .
\end{aligned}
$$

The metric tensor is transformed into the following:

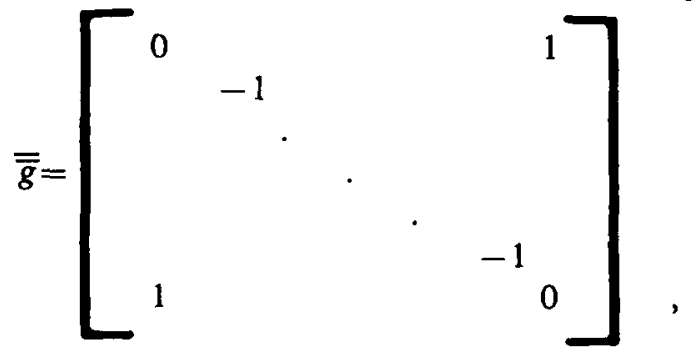

while the new $M_{\mu \nu}$ and $P_{\mu}$ are given by

$$
\overline{\bar{P}}_{\mu}=\left(\left(\bar{P}_{0}+\bar{P}_{n}\right) / \sqrt{2}, P_{1}, \ldots, P_{n-1},\left(\bar{P}_{0}-\bar{P}_{n}\right) / \sqrt{2}\right),
$$

$$
\left.\begin{array}{cc}
\cdots & K_{n} \\
\cdots & \left(K_{1}-M_{1 n}\right) / \sqrt{2} \\
\cdots & \left(K_{2}-M_{2 n}\right) / \sqrt{2} \\
& \vdots \\
& \cdot
\end{array}\right)
$$

which constitute the Lie algebra of the Galilei group in $(n-1)$ space/ 1 time dimensions.

\section{THE CONTRACTION $c \rightarrow \infty$ AS A COORDINATE TRANSFORMATION}

In this section we consider the case $n=4$ for simplicity, but all the results are immediately generalizable to arbitrary $n$. Let us start with the commutation relations (2.1) in the particular case $n=4$. Consider now the following coordinate transformation:

$$
\begin{aligned}
& \bar{x}^{0}=\alpha x^{0}+\beta x^{4}, \\
& \bar{x}^{i}=x^{i} \quad(i=1,2,3), \\
& \bar{x}^{4}=\gamma x^{0}+\delta x^{4} .
\end{aligned}
$$

The commutation relations for the generators

$$
\bar{l}_{i}=l_{i} \equiv-\frac{1}{2} \epsilon_{i j k} L_{j k}, \quad \bar{d}_{i}=d_{i}
$$




$$
\begin{gathered}
\bar{d}_{0}=\frac{\delta}{\alpha \delta-\beta \gamma} d_{0}-\frac{\gamma}{\alpha \delta-\beta \gamma} d_{4}, \\
k_{i} \equiv \frac{\overline{M^{0}}}{\alpha \delta}=-\left(\alpha g_{i}+\beta \lambda_{i}\right) \\
\left(i_{2}, k=1,2,3\right) \quad\left(\lambda_{i} \equiv L_{4 i}\right),
\end{gathered}
$$

are given by

$$
\begin{aligned}
& {\left[l_{i}, l_{j}\right]=i \epsilon_{i j k} l_{k}, \quad\left[l_{i}, k_{j}\right]=i \epsilon_{i j k} k_{k},} \\
& {\left[l_{i}, d_{j}\right]=i \epsilon_{i j k} d_{k}, \quad\left[k_{i}, k_{j}\right]=i b^{2} \epsilon_{i j k} l_{k},} \\
& {\left[d_{i}, d_{j}\right]=\left[l_{i}, h\right]=\left[d_{i}, h\right]=0,} \\
& {\left[k_{i}, h\right]=-i\left(\frac{\alpha \delta}{\alpha \delta-\beta \gamma}+\frac{\beta \gamma}{\alpha \delta-\beta \gamma}\right) d_{i},} \\
& {\left[k_{i}, d_{j}\right]=-i\left(\alpha \mu+\beta^{2} h+\beta \delta d_{4}\right) \delta_{i j} .}
\end{aligned}
$$

Depending on the values of the constants $\alpha, \beta, \gamma, \delta$, these relations either constitute the algebra of the Poincaré group or the algebra of the Galilei group. In fact, for

$$
\alpha=0, \quad \beta= \pm i, \quad \gamma \text { arbitrary, } \delta=0 .
$$

The relations (4.2) turn out to be the (3.1) in the case $n=3$, while for

$$
\alpha=1, \quad \beta=0, \quad \gamma \text { and } \delta \text { arbitrary. }
$$

Equations (4.2) are the same as (2.1) in the case $n=2$ (with $\left.g_{i} \equiv-k_{i}\right)$.

The coordinate transformation can be parametrized in order to include these two particular cases for different values of the parameter. In fact, the following transformation:

$\bar{x}=T(\epsilon, \gamma) x, \quad T(\epsilon, \gamma) \equiv\left(\begin{array}{ccc}\cosh i \epsilon & 0 & \text { sinhi } i \epsilon \\ 0 & I_{3} & 0 \\ \gamma & 0 & 0\end{array}\right)$.

reduces to (4.3) when $\epsilon= \pm \pi / 2$ and to (4.4) when $\epsilon=0$.

On the other hand, in order to compare this procedure with the usual contraction of the Poincare to the Galilei group when $c \rightarrow \infty$, we now confront (4.2) with the commutation relations obtained from the Poincare algebra after the application of the limiting process, but where the terms up to first order in $1 / c^{2}$ are still taken into account ${ }^{7}$ :

$$
\begin{aligned}
& {\left[L_{i}, L_{j}\right]=i \epsilon_{i j k} L_{k}, \quad\left[L_{i}, K_{j}^{\prime}\right]=i \epsilon_{i j k} K_{k}^{\prime},} \\
& {\left[L_{i}, P_{j}\right]=i \epsilon_{i j k} P_{k}, \quad\left[P_{i}, P_{j}\right]=\left[L_{i}, H^{\prime}\right]=\left[P_{i}, H^{\prime}\right]=0,} \\
& {\left[K_{i}^{\prime}, H^{\prime}\right]=i P_{i}, \quad\left[K_{i}^{\prime}, K_{j}^{\prime}\right]=-\left(i / c^{2}\right) \epsilon_{i j k} L_{k},} \\
& {\left[K_{i}^{\prime}, P_{j}\right]=\left(i / c^{2}\right) \delta_{i j} H^{\prime}+i \mu \delta_{i j}}
\end{aligned}
$$

We see that (4.6) can be obtained from (4.2) provided we put

$$
\alpha=1, \quad \beta= \pm i / c, \quad \gamma \text { arbitrary, } \delta=0 .
$$

This transformation is also obtained from (4.5) when $\epsilon= \pm 1 / c$. Notice that the arbitrarity of $\delta$ in (4.4) is only attained when $\epsilon=0$. Therefore it is not restrictive to put $\delta=0$, in general, as we have done in (4.5) to define the parametrization.

Within the parametrization (4.5) we have been able to reproduce the usual contraction $c \rightarrow \infty$, both when first-order terms in $1 / c^{2}$ are considered and also when the limit is fully applied. Observe that the full contraction corresponds to a finite jump from $\pm \pi / 2$ to 0 of the parameter $\epsilon$, while the supression of the terms of first order in $1 / c^{2}$ (i.e., the last step of the full contraction ) only amounts to a correspondingly infinitesimal change from $\epsilon= \pm 1 / c$ to $\epsilon=0$.

\section{CONCLUSIONS}

We have seen that Poincare algebra is a subalgebra of complex Galilei algebra while the Galilei algebra is a subalgebra of the ordinary Poincare algebra, if we consider them in arbitrary dimensional space. And this has been shown by means of very simple linear changes of coordinates: the lightcone transformation' in one case and an imaginary coordinate transformation ${ }^{5}$ in the other. The physical relevance of this result lies in the fact that, whatever be the invariance group of the physical theory that we take at the beginning (Poincare or Galilei) it is always possible to extract a theory, in a space with one dimension less, invariant under the other group.

Making use of a convenient parametrization of the imaginary transformation, ${ }^{5}$ we realized that the usual procedure of contraction of the Poincare to the Galilei group can be put into the form of a change of the space-time coordinates.

\section{ACKNOWLEDGMENT}

We would like to thank J.E.N. (Junta de Energía Nuclear, Spain) for financial support.

J.B. Kogut and D.E. Soper, Phys. Rev. D 1, 2901 ( 1970); J.D. Bjorken, J.B. Kogut, and D.E. Soper, Phys. Rev. D 3, 1382 (1971).

${ }_{2}^{2}$ J. Kogut and L. Susskind, Phys. Repts. 8, 75 (1973), see also original references therein.

${ }^{3}$ E. Elizalde and J. Gomis, Nuovo Cimento A 35, 336,347 (1976).

${ }^{4}$ E. Elizalde and J. Gomis, Nuovo Cimento A 35, 367 ( 1976); Nucl. Phys. B 122,535 (1977).

'E. Elizalde, "Poincaré is Subgroup of Galilei in One Space Dimension More," J. Math. Phys. 19, 526 (1978).

${ }^{6}$ E. Elizalde, "Interrelations between Relativistic and Galilean Field Equations for Arbitrary Spin Particles," University of Barcelona, preprint (1977).

'E.G. Sudarshan and N. Mukunda, Classical Dynamics (Wiley, New York, 1974). 